The treatment landscape in advanced melanoma is changing dramatically with the approval of new drugs. Vemurafenib was the first approved targeted agent for the treatment of BRAF-mutant advanced melanoma. However, treatment with a BRAF inhibitor is linked with acquired resistance occurring in half of the patients after approximately six months. Combination of MEK and BRAF inhibitor therapy results in extension of the time to resistance, translating into longer overall survival of treated patients. Similar clinical benefits are observed with therapy using antibodies against immune-checkpoint inhibitors in the same patient population. Due to the fact that results of randomised studies comparing these two treatment strategies back to back have not been presented yet, the best first and second line treatment option in patients with BRAF-mutant melanoma is unknown. Currently, phase 3 studies are also evaluating the efficacy of targeted therapy combined with immunotherapy in patients with BRAF-mutant and $B R A F$ wild-type advanced melanoma. Identifying a biomarker for the selection of patients benefiting most from the treatment will be crucial for further survival improvement in patients with advanced melanoma.

Key words: BRAF inhibitors, MEK inhibitors, melanoma.

Contemp Oncol (Pozn) 2018; 22 (1A): 68-72 DOI: https://doi.org/10.5114/wo.2018.73890

\section{BRAF and MEK inhibitors in the era of immunotherapy in melanoma patients}

\author{
Jacek Mackiewicz ${ }^{1-3}$, Andrzej Mackiewicz ${ }^{3,4}$ \\ ${ }^{1}$ Department of Medical and Experimental Oncology, Heliodor Swiecicki University \\ Hospital, Poznan University of Medical Sciences, Poland \\ ${ }^{2}$ Department of Biology and Environmental Studies, Poznan University of Medical \\ Sciences, Poland \\ ${ }^{3}$ Department of Diagnostics and Cancer Immunology, Greater Poland Cancer Centre, \\ Poznan, Poland \\ ${ }^{4}$ Chair of Medical Biotechnology, Poznan University of Medical Sciences, Poland
}

\section{Introduction}

The treatment landscape of advanced melanoma is changing together with the marketing authorisation of new medicinal products. Seven new drugs and three combinations of these drugs have been approved in the treatment of melanoma since the year 2010. Currently, in patients with BRAF wild-type (wt) advanced melanoma, anti-PD1 (programmed cell death factor 1) therapy with nivolumab or pembrolizumab is the standard of care. The combination of nivolumab and ipilimumab (anti-CTLA4 antibody) is also an option in patients with advanced non-resectable melanoma, but the treatment toxicity is very significant. In patients with BRAF mutation the above immunotherapy strategies can also be applied. High clinical efficacy is also observed in advanced melanoma patients with BRAF mutants using BRAF plus MEK inhibitors. Currently phase 3 studies are comparing the efficacy of immunotherapy and targeted therapy in patients with BRAF mutation.

\section{Genetic subtypes of melanoma}

Activating mutation of the serine-threonine kinase BRAF gene is the most frequent genetic alteration in melanomas. BRAF mutation is observed in about $50 \%$ of skin melanoma and in $10-20 \%$ of mucosal melanoma cases [1-4]. Mutation in BRAF gene activates BRAF protein, which increases proliferation and survival of melanoma cells [5]. Most frequently (in about $90 \%$ of cases) valine is substituted with glutamate in the 600 codon (V600E), less frequently with lysine (V600K) or arginine (V600R) [1, 2].

The second most frequent genetic alteration is RAS mutation, observed in $25 \%$ of melanomas. The most commonly seen is NRAS mutation [2, 4, 6]. Mutations in the RAS gene keep RAS protein in the active state, which activates RAF and subsequently MEK and ERK, leading to activation of the MAPK signalling pathway. RAS can also activate other pathways such as the PI3K (phosphatidylinositol-3 kinase) pathway [7]. BRAF and NRAS are mutually exclusive.

Another frequently observed aberration (14\%) is the mutation in the NF1 (neurofibroma factor 1) gene. NF1 regulates RAS through GTP-ase activating protein. Due to the mutation in the NF1 gene, NF1 protein loses regulative properties leading to continuous activation of RAS [8]. The NF1 mutation is observed in $46 \%$ of BRAF-mutant and NRAS wild-type melanoma cases [3].

The triple-wild-type melanomas do not carry any of the mentioned mutations (BRAF, NRAS, NF1). This subgroup is linked instead with GNAQ (observed frequently in uveal melanoma) or KIT mutations [2]. 


\section{BRAF inhibitors}

Currently two BRAF inhibitors are approved in Europe and US for the treatment of patients with BRAF-mutant advanced melanoma - vemurafenib and dabrafenib. Encorafenib is the next highly explored BRAF inhibitor. These drugs are orally bioavailable, ATP-competitive, small-molecule inhibitors of BRAF kinase.

In a randomised phase 3 study (BRIM-3) patients receiving vemurafenib demonstrated a higher response rate compared to those treated with dacarbazine (DTIC) - 57\% vs. $9 \%$. The median progression-free survival (PFS) in the study group treated with vemurafenib was significantly longer -6.9 vs. 1.6 months (HR 0.38; $p<0.0001)$. Patients treated with vemurafenib also presented longer median overall survival (OS) compared to the control group - 13.6 vs. 9.7 months (HR 0.70; $p=0.0008$ ) $[9,10]$.

The most frequently observed adverse events (AEs) in patients treated with vemurafenib are arthralgia (56\%), fatigue (46\%), rash (41\%), and photosensitivity (41\%). The highest frequency of grade 3 and 4 toxicity is cutaneous squamous cell carcinoma (SCC) (19\%), keratoacanthoma (10\%), rash (9\%) and elevated aminotransferases (11\%) [11].

Dabrafenib was approved in advanced melanoma following the results of a randomised phase 3 study (BREAK-3) in patients with BRAF mutation. This trial demonstrated similar results to the BRIM-3 study. The response rate in patients treated in the dabrafenib group was higher compared to the DTIC group - 50\% vs 6\%. The median PFS in patients receiving dabrafenib was 6.9 months and 2.7 months in patients treated with DTIC (HR $0.37 ; p<0.0001)$. The median OS at the last study update was 18.2 months in the dabrafenib group and 15.6 months in the DTIC group (HR 0.76) [12, 13].

Treatment with dabrafenib was associated with hyperkeratosis (36\%), rash (30\%), alopecia (27\%), skin papilloma (22\%), palmar-plantar hyperkeratosis (19\%), arthralgia (19\%), fatigue (18\%), and headache (18\%). The most frequently observed grade 3 and 4 adverse events were cutaneous SCC (7\%) and pyrexia (3\%) $[12,13]$.

It is difficult to compare the toxicity of vemurafenib and dabrafenib. However, in patients treated with vemurafenib higher frequency of photosensitivity (dabrafenib-2\%) and SCC/keratoacanthoma was observed. In patients receiving dabrafenib pyrexia is more frequently documented.

\section{MEK inhibitors}

MEK inhibitors are bioavailable, non-ATP competitive, allosteric binding inhibitors of MEK. Cobimetinib is a MEK1 inhibitor, while trametinib and binimetinib inhibit both MEK1 and MEK2.

In a phase 3 study trametinib demonstrated increased median PFS compared to DTIC (4.8 vs. 1.5 months, HR 0.45, $p<0.001)$ in patients with advanced BRAF-mutant melanoma. Also the 6-month OS was higher in patients receiving trametinib (81\% vs. 67\%, $p=0.01$ ) [14].

In a phase 2 study binimetinib was evaluated in patients with advanced melanoma harbouring BRAF or NRAS mutation. The response rate was $20 \%$ in both groups with similar median PFS (3.6 months - BRAF-mutant, 3.7 months
- NRAS-mutant) [15]. Binimetinib was also evaluated in a phase 3 study (NEMO) in patients with NRAS mutation. The median PFS in patients treated with binimetinib was 2.8 months compared to 1.5 months in the group treated with DTIC (HR 0.62; $p<0.001)[16]$. MEK inhibitors present a different toxicity profile than BRAF inhibitors. The most frequently observed $A E$ is rash, observed in $57 \%$ of patients. MEK inhibitors cause papulopustular rash, while BRAF inhibitors cause hyperkeratotic maculopapular rash. Other frequently observed MEK inhibitor related AEs include diarrhoea (43\%) and peripheral oedema (26\%). The most frequently noted grade 3 and 4 AEs are hypertension (12\%), rash (8\%) and fatigue (4\%). Other AEs also noted included nausea, alopecia, constipation, vomiting, reduction of left ventricular ejection fraction (LVEF) and ocular toxicity (blurred vision, reversible chorioretinopathy) [17, 18].

\section{BRAF plus MEK inhibitors}

Treatment with BRAF inhibitors is associated with acquired resistance after an earlier response. Half of the patients developed progression of the disease after approximately 6 months of treatment. It has been shown that addition of MEK inhibitor to BRAF inhibitor therapy may break the treatment resistance in preclinical studies [19].

In a randomised phase 3 study (COMBI-d) the combination of dabrafenib with trametinib showed a higher response rate compared to dabrafenib alone in patients with advanced BRAF-mutant melanoma - 69\% vs. 53\% $(p=0.0014)$. The median PFS was also longer in patients receiving the combination therapy (11.0 vs. 8.8 months; $\mathrm{HR}=0.67 ; p=0.0004)$. The median OS in patients treated with dabrafenib combined with trametinib was 25.1 compared to 18.7 months in patients receiving dabrafenib monotherapy ( $\mathrm{HR}=0.71 ; p=0.0107)$ [20]. In a longer follow-up 3-year survival in patients treated with the combination was observed in $44 \%$ of patients. [21]

Similar results were observed in another phase 3 study (COMBI-v) where dabrafenib combined with trametinib was compared with vemurafenib, showing a higher response rate in patients receiving two drugs - $64 \%$ vs. $51 \%$ $(p<0.001)$. The median PFS was longer in the group treated with dabrafenib plus trametinib compared to the vemurafenib group (11.4 vs. 7.3 months; HR 0.56; $p<0.001$ ) [22]. At the European Society of Medical Oncology (ESMO) 2016 congress updated survival results from this study were presented. In patients receiving dabrafenib plus trametinib the median OS was 26.1 months, while in the group treated with vemurafenib it was 17.8 months $(H R=0.68)$, with 3 -year OS in $45 \%$ and $31 \%$ of patients respectively [23]. The evaluated quality of life was significantly better in the combination group [24].

In a phase 3 study (CoBRIM) the efficacy of vemurafenib combined with cobimetinib (MEK inhibitor) was compared with vemurafenib alone. Patients treated with the combination of BRAF and MEK inhibitor demonstrated a higher response rate than in the BRAF inhibitor monotherapy group $-68 \%$ vs. $45 \%(p<0.001)$. The median PFS was longer in patients receiving the combination - 9.9 vs. 6.2 months $(H R=0.51 ; p<0.001)$. The median OS in patients 
treated with the combination of vemurafenib and cobimetinib was 22.3 months, while in the group receiving vemurafenib alone it was 17.4 months $(\mathrm{HR}=0.70 ; p=0.005)$ [25]. At the Society for Melanoma Research (SMR) 2016 congress updated survival results from this study showed 3 -year OS in $37.4 \%$ of patients treated with the combination and $31.1 \%$ receiving vemurafenib alone. These differences in survival between the COMBI-v and coBRIM study might be associated with higher frequency of patients with elevated serum LDH in the coBRIM study (46\% vs. $34 \%$ ) [26]. Elevated LDH is a poor prognostic factor regardless of the treatment used [21, 26-28].

In a phase 1 b study (BRIM7) in 63 treatment naïve patients receiving vemurafenib combined with cobimetinib the median OS was 31.2 months, while 3- and 4-year survival was $39.2 \%$ and $35.9 \%$ respectively [26].

Another interesting molecule is encorafenib, a BRAF inhibitor with higher affinity to BRAF and extended binding time. Encorafenib was evaluated in combination with binimetinib (MEK inhibitor) in a phase 3 study and demonstrated longer median PFS (14.9 months) compared to vemurafenib alone (7.3 months) with HR of 0.54 ( $p<0.001)$. In the third group of patients receiving encorafenib monotherapy the median PFS was 9.6 months with HR of 0.75 However, the difference as compared to encorafenib plus binimetinib was not statistically significant $(p=0.051)[29]$.

\section{Elevated lactate dehydrogenase}

It has been shown that melanoma patients with elevated serum LDH treated with immune checkpoint inhibitors present a poor prognosis $[27,28]$.

In the COMBI-d study $25 \%$ of patients with elevated and $52 \%$ with normal LDH level treated with dabrafenib plus trametinib survived 3 years. The best 3 -year survival time (62\%) was noted in patients with normal LDH and less then 3 metastatic sites. Patients presenting elevated $\mathrm{LDH}$ and treated with vemurafenib in the control arm also presented a worse outcome compared to those with normal LDH. Patients with elevated LDH treated with vemurafenib and cobimetinib in the coBRIM study also had a worse prognosis (3-year survival: $22.8 \%$ ) compared to patients with normal LDH (3-year survival: 47.8\%) [26].

\section{Combination of targeted therapy with immune checkpoint inhibitors}

Various preclinical studies showed increased efficacy of targeted therapy combined with immune checkpoint inhibitors as compared to monotherapy with these drugs [30]. An early phase clinical study evaluating the combination of vemurafenib with ipilimumab revealed unexpected toxicity in patients with advanced melanoma. The observed hepatotoxicity occurred within the first 2 to 5 weeks of treatment in 6 out of 10 patients and required discontinuation of the study [31]. In another study dabrafenib +/- trametinib was combined with ipilimumab, but due to increased incidence of colitis with perforation of the colon the accrual of patients was subsequently terminated [32].
The high toxicity of BRAF/MEK inhibitors combined with ipilimumab and the parallel development of antibodies targeting PD1 and PDL-1 lead to the alternative strategy of exploring the combination of anti-PD1/PD-L1 with targeted therapy.

A phase 1 study evaluating the combination of anti-PD-L1 (MEDI4736) with dabrafenib and/or trametinib in BRAF-mutant and BRAF wild-type melanoma patients was carried out. In the 26 patients with $B R A F$ mutation receiving the combination of MEDI4736, dabrafenib and trametinib the response rate (RR) was 69\% and the disease control rate $(D C R=C R+P R+S D)$ was $79 \%$. In 20 patients with BRAF wild-type melanoma treated with trametinib and MEDI4736 the RR was $21 \%$ and DCR was $80 \%$. In the last BRAF wild-type group the 19 patients were receiving trametinib monotherapy with subsequent administration of trametinib and MEDI4736, followed by MEDI4736 monotherapy $(R R=21 \%$; $D C R=80 \%)$. However, the treatment demonstrated high toxicity. The frequency of treatment-related AE depended on the study group. Any grade AEs were observed in $85-100 \%$, and grade 3 and 4 AEs were noted in 17-40\% of patients [33].

At the SMR 2016 Congress results from a phase 1b study evaluating the combination of atezolizumab (anti-PD-L1) with vemurafenib and cobimetinib were presented. Only 29 BRAF-mutant advanced melanoma patients were evaluated. The RR was $83 \%$ (CR - 10\%, PR - 72\%) with stable disease (SD) observed in 10\%. Only 3\% of patients developed progression of the disease. The treatment benefit was, however, associated with high toxicity. All grades of treatment-related AEs were seen in all patients, while grades 3 and 4 AEs were noted in 40\% of patients [34]. Currently the phase 3 study is ongoing [35]. Results of a phase $1 b$ study in patients with $B R A F$ wild-type melanoma receiving the combination of atezolizumab together with trametinib demonstrated an RR of 50\%, with high toxicity. However in this cohort only 10 patients were evaluated [36]. A phase 3 study in this group of patients is planned shortly.

An interesting phase 3 study evaluating the efficacy of combination of PDR001 (anti-PD1) with dabrafenib and trametinib compared to dabrafenib and trametinib alone is starting accrual in the near future [37]. A phase 2 study evaluating the combination of pembrolizumab with dabrafenib and trametinib in patients with $B R A F$-mutant advanced melanoma is about to be completed. In another cohort BRAF wild-type melanoma patients are receiving pembrolizumab combined with trametinib (concurrent or intermittent dosing) [38]

\section{Conclusions}

The combination of BRAF and MEK inhibitors shows very high activity in advanced melanoma patients with the BRAF mutation. Currently, there is a lack of phase 3 study results comparing the efficacy of targeted therapy and immune checkpoint inhibitors in patients harbouring the BRAF mutation; therefore the treatment decision for the physicians in this patient population is very difficult. The combination of targeted therapy with immune checkpoint inhibitors shows promising results in early phase 
trials, but requires validation in ongoing phase 3 studies [34, 36, 39, 40]. Novel treatment strategies in preclinical melanoma studies are promising. The combination of cancer vaccines with anti-PD1 antibodies shows a synergistic effect [41]. Moreover, agents targeting cancer metabolism enhance the activity of anti-PD1 therapy. Another important discovery is that the patient gut microbiome may enhance anti-PD1 treatment. The promising early results of combinatorial treatment strategies bring hope for further progress in the management of patients with advanced melanoma. The most expected are the results of the adjuvant treatment trials of advanced melanoma patients with resected metastases both of targeted strategies and various immunotherapeutic approaches.

The authors declare no conflict of interest.

\section{References}

1. Sullivan R, LoRusso P, Boerner S, Dummer R. Achievements and challenges of molecular targeted therapy in melanoma. Am Soc Clin Oncol Educ Book 2015; 177-86.

2. Akbani R, Akdemir KC, Aksoy BA et al. Genomic classification of cutaneous melanoma. Cell 2015; 161: 1681-96.

3. Krauthammer $M$, Kong $Y$, Bacchiocchi $A$, et al. Exome sequencing identifies recurrent mutations in NF1 and RASopathy genes in sunexposed melanomas. Nat Genet 2015; 47: 996-1002.

4. Sosman JA, Puzanov I. Molecular targets in melanoma from angiogenesis to apoptosis. Clin Cancer Res 2006; 12: 2376s-83s.

5. Jovanovic B, Krockel D, Linden D, Nilsson B, Egyhazi S, Hansson J. Lack of cytoplasmic ERK activation is an independent adverse prognostic factor in primary cutaneous melanoma. I Invest Dermatol 2008; 128: 2696-704.

6. Stephen AG, Esposito D, Bagni RK, McCormick F. Dragging ras back in the ring. Cancer Cell 2014; 25: 272-281.

7. Mandala M, Merelli B, Massi D. Nras in melanoma: targeting the undruggable target. Crit Rev Oncol Hematol 2014; 92: 107-22.

8. Sullivan RJ. The role of mitogen-activated protein targeting in melanoma beyond BRAFV600. Curr Opin Oncol 2016; 28:185-91.

9. Chapman PB, Hauschild A, Robert C, et al. Improved survival with vemurafenib in melanoma with BRAF V600E mutation. N Engl J Med 2011; 364: 2507-16.

10. McArthur GA, Chapman PB, Robert C, et al. Safety and efficacy of vemurafenib in BRAF(V600E) and BRAF(V600K) mutation-positive melanoma (BRIM-3): extended follow-up of a phase 3, randomised, open-label study. Lancet Oncol 2014; 15: 323-32.

11. Zimmer L, Hillen U, Livingstone E, et al. Atypical melanocytic proliferations and new primary melanomas in patients with advanced melanoma undergoing selective BRAF inhibition. J Clin Oncol 2012; 30: 2375-83.

12. Hauschild A, Grob JJ, Demidov LV, et al. Dabrafenib in BRAFmutated metastatic melanoma: a multicentre, open-label, phase 3 randomised controlled trial. Lancet 2012; 380: 358-65.

13. Hauschild A, Grob JJ, Demidov LV, et al. An update on BREAK-3, a phase III, randomized trial: dabrafenib (DAB) versus dacarbazine (DTIC) in patients with BRAF V600E-positive mutation metastatic melanoma (MM). American Society of Clinical Oncology Annual Meeting, Chicago, USA. Abstracts 31 (15_suppl):9013.

14. Flaherty KT, Robert C, Hersey P, et al. Improved survival with MEK inhibition in BRAF-mutated melanoma. N Engl J Med 2012; 367: 107-14

15. Ascierto PA, Schadendorf D, Berking C, et al. MEK162 for patients with advanced melanoma harbouring NRAS or Val600 BRAF mutations: a non-randomised, open-label phase 2 study. Lancet Oncol 2013; 14: 249-56.

16. Dummer R, Schadendorf D, Ascierto PA, et al. Results of NEMO: a phase III trial of binimetinib (BINI) vs dacarbazine (DTIC) in NRAS mutant cutaneous melanoma. American Society of Clinical Oncology Annual Meeting, Chicago, USA. Abstract 34 (15_suppl): 9500.
17. Urner-Bloch U, Urner M, Stieger P, et al. Transient MEK inhibitor associated retinopathy in metastatic melanoma. Ann Oncol 2014; 25: 1437-41.

18. Schad K, Baumann Conzett K, Zipser MC, et al. Mitogen-activated protein/extracellular signal-regulated kinase kinase inhibition results in biphasic alteration of epidermal homeostasis with keratinocytic apoptosis and pigmentation disorders. Clin Cancer Res 2010; 16: 1058-64.

19. Villanueva J, Vultur A, Lee JT, Somasundaram R, et al. Acquired resistance to BRAF inhibitors mediated by a RAF kinase switch in melanoma can be overcome by cotargeting MEK and IGF-1R/PI3K. Cancer Cell 2010;18: 683-95.

20. Long GV, Stroyakovskiy D, Gogas H, et al. Dabrafenib and trametinib versus dabrafenib and placebo for Val600 BRAF-mutant melanoma: a multicentre, double-blind, phase 3 randomised controlled trial. Lancet 2015; 386: 444-51.

21. Flaherty K, Davies MA, Grob J), et al. Genomic analysis and 3-y efficacy and safety update of COMBI-d: a phase 3 study of dabrafenib (D) fl trametinib (T) vs D monotherapy in patients (pts) with unresectable or metastatic BRAF V600E/K-mutant cutaneous melanoma. American Society of Clinical Oncology Annual Meeting, Chicago, USA. Abstract 34 (15 suppl): 9502.

22. Robert C, Karaszewska B, Schachter J, et al. Improved overall survival in melanoma with combined dabrafenib and trametinib. N Engl J Med 2015; 372: 30-9.

23. Robert C, Karaszewska B, J. Schachter, et al. Three-year estimate of overall survival in COMBI-v, a randomized phase 3 study evaluating first-line dabrafenib (D) + trametinib (T) in patients (pts) with unresectable or metastatic BRAF V600E/K-mutant cutaneous melanoma. Ann Oncol 2016; 27: 1-36.

24. Grob JJ, Amonkar MM, Karaszewska B, et al. Comparison of dabrafenib and trametinib combination therapy with vemurafenib monotherapy on health-related quality of life in patients with unresectable or metastatic cutaneous BRAF Val600-mutation-positive melanoma (COMBI-v): results of a phase 3 , open-label, randomized trial. Lancet Oncol 2015; 16: 1389-98.

25. Larkin J, Ascierto PA, Dreno B, et al. Combined vemurafenib and cobimetinib in BRAF-mutated melanoma. N Engl J Med 2014; 371 : 1867-76.

26. McArthur GA, Dréno B, Atkinson V, et al. Efficacy of Long-Term Cobimetinib + Vemurafenib in Advanced BRAF ${ }^{\mathrm{V} 600}$-Mutated Melanoma: 3-Year Follow-up of coBRIM (Phase 3) and 4-year Follow-Up of BRIM7 (Phase 1b). Society for Melanoma Research 2016 Congress; November 6-9, 2016; Boston, MA, USA.

27. Kelderman S, Heemskerk B, van Tinteren $\mathrm{H}$, et al. Lactate dehydrogenase as a selection criteria for ipilimumab treatment in metastatic melanoma. Cancer Immunol Immunother 2014; 63: 449-58.

28. Larkin J, Ferrucci PF, Gonzalez R, et al. Efficacy of Nivolumab Plus Ipilimumab Combination in Patients With Advanced Melanoma and Elevated Serum Lactate Dehydrogenase: A Pooled Analysis. $13^{\text {th }}$ International Congress of the Society for Melanoma Research 2016, November 6-9, 2016, Boston, USA.

29. Dummer R, Ascierto PA, Gogas HJ, et al. Results of COLUMBUS Part 1: A Phase 3 Trial of Encorafenib (ENCO) Plus Binimetinib (BINI) Versus Vemurafenib (VEM) or ENCO in BRAF-Mutant Melanoma. $13^{\text {th }}$ International Congress of the Society for Melanoma Research 2016, November 6-9, 2016, Boston, USA.

30. Liu L, Mayes PA, Eastman S, et al. The BRAF and MEK inhibitors dabrafenib and trametinib: effects on immune function and in combination with immunomodulatory antibodies targeting PD-1, PD-L1, and CTLA-4. Clinical Cancer Research 2015; 21: 1639-51.

31. Ribas A, Hodi FS, Callahan M, Konto C, Wolchock J. Hepatotoxicity with the combination of vemurafenib and ipilimumab. N Engl J Med 2013; 368: 1365-6.

32. Puzanov I. Combining targeted and immunotherapy: BRAF inhibitor dabrafenib (D) +/- the MEK inhibitor trametinib (T) in combination with ipilimumab (Ipi) for V600E/K mutation positive unresectable or metastatic melanoma (MM). Journal of Translational Medicine 2015; 13 (suppl 1), K8.

33. Ribas A, Butler M, Lutzky J, et al. Phase 1 study combining anti-PD-L1 (MEDI4736) with BRAF (dabrafenib) and/or MEK (trametinib) inhibitors in advanced melanoma. J Clin Oncol 2015; 33: 3003. 
34. Sullivan R, Hamid O, Gonzalez R, et al. Safety and clinical activity of atezolizumab + cobimetinib + vemurafenib in BRAFV600-mutant metastatic melanoma. $13^{\text {th }}$ International Congress of the Society for Melanoma Research 2016, November 6-9, 2016, Boston, USA.

35. clinicaltrials.gov; NCT02908672

36. Infante J, Kim TM, Friedmann J, et al. Safety and clinical activity of atezolizumab combined with cobimetinib in metastatic melanoma. $13^{\text {th }}$ International Congress of the Society for Melanoma Research 2016, November 6-9, 2016, Boston, USA.

37. clinicaltrials.gov; NCT02967692

38. clinicaltrials.gov; NCT02130466

39. clinicaltrials.gov; NCT02908672

40. clinicaltrials.gov; NCT03178851

41. Karaki S, Anson M, Tran T, Giusti D, Blanc C, Oudard S, Tartour E. Is there still room for cancer vaccines at the era of checkpoint inhibitors. Vaccines (Basel) 2016; 4: pii: E37.

\section{Address for correspondence}

\section{Jacek Mackiewicz}

Department of Medical and Experimental Oncology

Heliodor Swiecicki University Hospital

Poznan University of Medical Sciences

Grunwaldzka 16/18

Poznan, Poland

e-mail: jmackiewicz@biocontract.com 